\title{
ORIGINAL RESEARCH \\ Topographic Analysis of the Inferior Parietal Lobule in High-Resolution 3D MR Imaging
}

I. Kiriyama

H. Miki

K. Kikuchi

S. Ohue

S. Matsuda

T. Mochizuki
BACKGROUND AND PURPOSE: Recent functional MR imaging studies have revealed that the inferior parietal lobule (IPL) supports numerous functions; however, the precise organization of the IPL remains unclear. It has been previously reported that the individual components of the IPL show variable morphologic features; thus, any investigations of IPL function must take into account the variable topography of the IPL. However, it is difficult to understand the topography of the IPL only in transaxial and/or sagittal MR images. We generated volume rendering (VR) images of the brain from high-resolution 3D MR imaging data and analyzed the topography of the IPL.

MATERIALS AND METHODS: Forty healthy volunteers were examined with 1.5T MR imaging, and VR images were generated. Sulcal continuity, sulcal connections, and the gyral pattern of the IPL were investigated.

RESULTS: The main sulci and gyri were able to be identified excellently on VR images of all volunteers. One long, continuous postcentral sulcus (postCS) was seen in $47.5 \%$ and $40 \%$ in the right and left hemisphere, respectively. The frequency with which the postCS connected with the anterior end of the IPS was $72.5 \%$ and $75 \%$ in the right and left, respectively. The gyral pattern of the IPL showed variable morphology.

CoNCLUSIONS: VR images can depict the sulcal continuity and connections of the IPL accurately. VR imaging is a very useful noninvasive technique to observe the topography of the IPL and should contribute to the investigation of functional localization. $t$ is well known that the topography of the brain correlates with specific neurologic functions. The inferior parietal lobule (IPL) supports an extensive range of functions. In the classic sense, it was known that Gerstmann syndrome (finger agnosia, right/left disorientation, acalculia, and agraphia) was associated with the IPL. ${ }^{1-3}$ Furthermore, recent functional MR imaging studies have revealed that the IPL supports spatial representation, multimodal integration, attentional control, numeric judgments, motor planning, working memory, and numerous other critical functions. ${ }^{4-7}$ However, the precise organization of the IPL remains unclear. In regard to the anatomy, some investigators have reported that the individual components of the IPL show variable morphologic features and marked right-left asymmetry. ${ }^{8-10}$ Thus, any investigations of IPL function must take into account the variable topography of the IPL.

However, it is difficult to identify the main sulci and gyri of the IPL, and it is more difficult to understand the topography of the entire IPL with use of only 2D transaxial and/or sagittal images. Investigations concerning the normal topography of the IPL with 2D MR imaging data are few, and those that have been performed have had limited descriptions of the IPL's sulcal connections. ${ }^{11,12}$ Advances in medical workstations in recent years have facilitated making 3D images of the brain and have enabled the noninvasive observation of individual brain topography. To the best of our knowledge, there have been no reports of the topography of the IPL using 3D MR

Received September 17, 2008; accepted after revision October 20.

From the Departments of Radiology (I.K., H.M., K.K., T.M.), Neurosurgery (S.O.), and Anatomy and Embryology (S.M.); Ehime University Graduate School of Medicine, Ehime, Japan.

Please address correspondence to Ikuko Kiriyama, Department of Radiology, Ehime University School of Medicine, Shitsukawa, Toon-City, Ehime, 791-0295 Japan; e-mail: ikukosan@m.ehime-u.ac.jp

DOl 10.3174/ajnr.A1417 imaging. Volume rendering (VR) is a method of constructing 3D images; VR of brain MR imaging can depict important landmarks of brain anatomy, which can be difficult to identify on cross-sectional images. ${ }^{13,14}$ We have investigated the optimal way to make high-resolution VR images for observation of the sulci and gyri. ${ }^{15}$ In this study, we report the sulcal continuity, sulcal connections, and gyral pattern of the IPL using VR images generated from high-resolution 3D MR imaging data and compare the results with previous cadaver studies to examine the accuracy of the VR images.

\section{Anatomy of the IPL $L^{8,10,16}$}

Figure 1 (left) shows the left hemisphere with use of a VR image. The central sulcus (CS) is the most important and constant landmark on the convexity of the brain. The CS extends obliquely across the lateral convex surface of the hemisphere and is generally continuous. It separates the frontal and parietal lobes and defines the anterior surface of the postcentral gyrus (postCG). The IPL is located posterior to the postCG and is the inferior component of the parietal lobe. Figure 1 (right) shows the main sulci and gyri of the IPL. The postcentral sulcus (postCS) forms the anterior border of the IPL. The postCS courses parallel with the CS and separates the postCG from the superior and the inferior parietal lobules behind it. The intraparietal sulcus (IPS) forms the superior and posterior border of the IPL. The IPS courses almost horizontally behind the CS and divides the convexity of the parietal lobe into superior and inferior parietal lobules. The IPS descends in the posterior and shifts to the intraoccipital sulcus. The horseshoe-shaped gyrus that surmounts the posterior ascending ramus of the Sylvian fissure (Sy) is the supramarginal gyrus (SMG), and it is the anterior portion of the IPL. The posterior ascending ramus of the Sy forms the posterior superior end of the Sy. The horseshoe-shaped gyrus that surmounts the pos- 

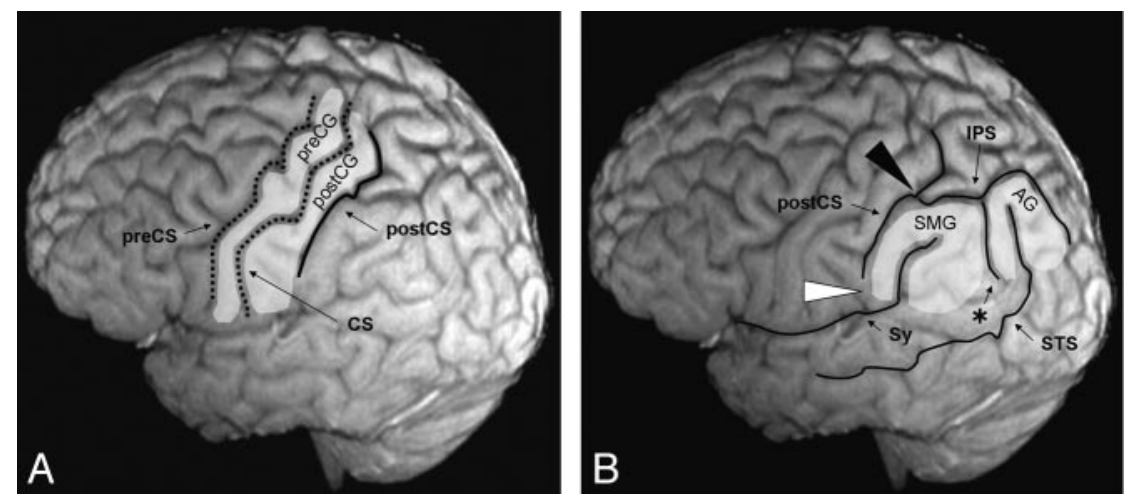

Fig 1. VR image of the left hemisphere of a healthy volunteer. The left image and the right image are the same. The IPL locates posterior to the postCG and is the inferior component of the parietal lobe. The postCS and the IPS form the border of the IPL. The postCS connects with the anterior end of the IPS (black arrowhead) and does not connect with the posterior ascending ramus of the Sy (white arrowhead) in this case. The asterisk indicates the sulcus intermedius primus.

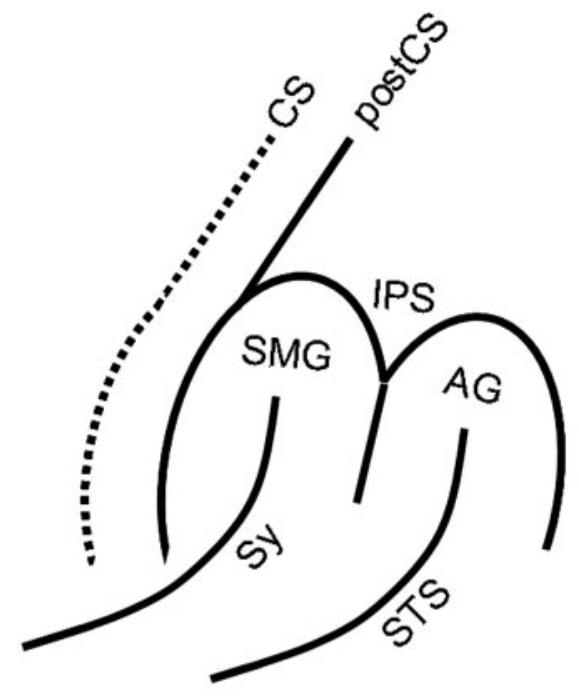

A
B

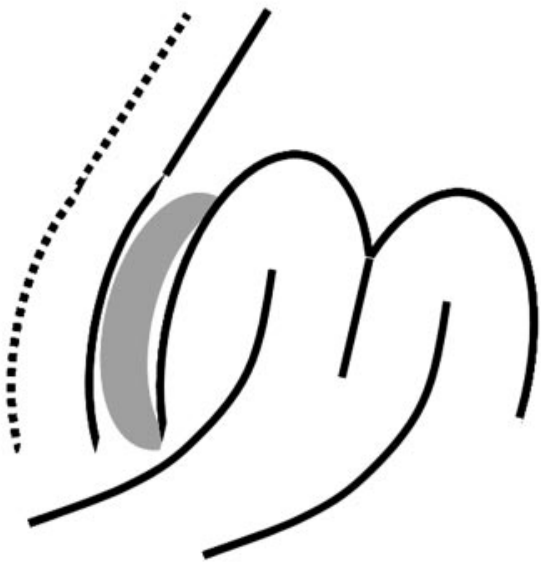

preSMG

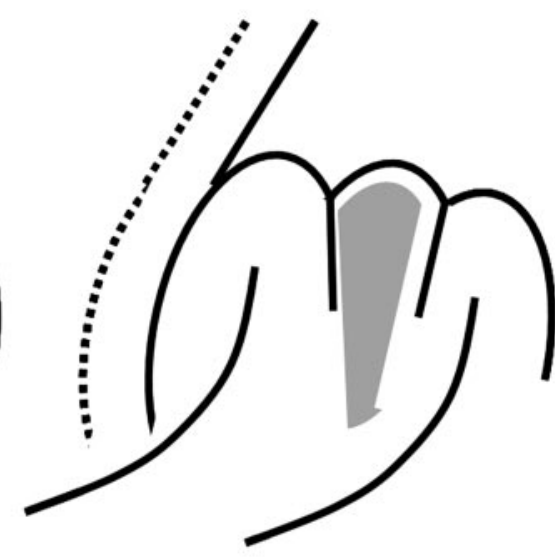

C
preAG

Fig 2. Patterns of the IPL. A, Typical pattern. There are no additional gyri. B, PreSMG pattern. The additional gyri (gray tissue) intercalated between the postCS and the SMG. $C$, PreAG. The additional gyri (gray tissue) positioned between the SMG and the AG.

terior end of the superior temporal sulcus (STS) is the angular gyrus (AG), and it is the posterior component of the IPL. The STS courses posteriorly and then superiorly in parallel with the Sy. The sulcus intermedius primus courses immediately posterior to the SMG, defining its posterior border.

\section{Materials and Methods}

Forty healthy volunteers (26 men and 14 women; age range, 21-47 years; mean age, 26.8 years) were examined with 1.5T MR imaging (Signa Excite; GE Medical Systems, Milwaukee, Wis) with an 8channel head coil. Written informed consent was obtained from all volunteers, and the study received the approval of the ethics committee of our institution. T1-weighted 3D-spoiled gradient-echo (SPGR) images were obtained in 5 minutes 11 seconds with TR, $25 \mathrm{~ms}$; TE, $2.5 \mathrm{~ms}$; flip angle, $20^{\circ}$; reduction factor, 2.0 ; matrix size, $192 \times 256$ (interpolated to $512 \times 512$ ); section thickness, $1.6 \mathrm{~mm}$; and slab, $1.6 \times 128$ (interpolated to $0.8 \times 256) \mathrm{mm} .{ }^{15} \mathrm{VR}$ images were generated from high-resolution $3 \mathrm{D}$ data on a workstation (Advantage
Workstation 4.1; GE Medical Systems) with black-and-white shading. The CSF space was identified semiautomatically by the signal intensity, and the tissues on the outside of the CSF space were removed. Identification of the sulci and gyri was performed with VR images on a monitor.

Initially, we examined the sulcal continuity of the postCS and IPS, the sulcal connections of the postCS, the anterior end of the IPS (Fig $1 B$, black arrowhead), and the sulcal connections of the inferior end of the postCS and the posterior ascending ramus of the Sy (Fig $1 B$, white arrowhead). Next, we investigated the gyral patterns of the IPL. We tried to classify the IPL into 4 patterns (typical, preSMG, preAG, and others) according to the gyral arrangement and referring to previous reports of Naidich et $\mathrm{al}^{10}$ in the classification of IPL. Figure 2 shows the schema of IPL patterns. Typical patterns were defined as those in which the SMG and AG were located behind the postCS, those in which additional gyri were intercalated between the postCS and the SMG were defined as preSMG, and those cases in which the additional gyri were positioned between the SMG and AG were de- 
fined as preAG. Using a similar approach, we also classified an additional 20 fixed specimens of whole-body donors for anatomic dissection ( 8 men and 12 women) for comparison with the VR images.

\section{Results}

Table 1 shows results from our studies of sulcal continuity. A single continuous postCS was observed on the right in $47.5 \%$ and on the left in $40 \%$ of our cases. A single long IPS was observed on the right and left in $65 \%$ and $70 \%$ of our cases, respectively.

Table 2 shows the sulcal connections of the postCS and the anterior end of the IPS, and the posterior ascending ramus of the Sy. The frequency with which the postCS connected with the anterior end of the IPS was $72.5 \%$ and $75 \%$ in the right and left hemispheres, respectively. The frequency with which the postCS connected with the Sy differed between the right and left sides (52.5\% and 30\%, respectively).

Table 3 shows the frequency of the gyral pattern of the IPL, and Figure 3 shows each pattern according to VR images and specimens. A typical pattern was observed in only $42.5 \%$ of cases on the right and in 35\% of cases on the left with use of VR images, and in $45 \%$ of cases on the right and in $20 \%$ of cases on the left in specimens. A preSMG pattern was seen in only $2.5 \%$ of cases on the right side with use of VR images. No preAG pattern was seen on the left side with VR images, and $37.5 \%$ of our cases on the right and $47.5 \%$ of our cases on the left showed no discernible pattern with VR images, compared with $30 \%$ of cases on the right and $45 \%$ of cases on the left in specimens.

\section{Discussion}

The identification of the end of the postCS was comparatively easy, and the results of our studies of the sulcal continuity of the postCS demonstrated agreement with those of Ono et $\mathrm{al}^{8}$ with postmortem specimens. In contrast, there were some differences between the sulcal continuity of the IPS and those reported by Ono et al. ${ }^{8}$ In our study, the IPS was 1 continuous sulcus in the right hemisphere in $65 \%$ of our cases compared with 2 discontinuous segments as described in $68 \%$ of cases by Ono et al. ${ }^{8}$ One possible cause for this difference is difficulty in identifying the end of the IPS. Duvernoy ${ }^{16}$ divided the IPS into 3 parts (ascending, horizontal, and descending), and he concluded that the postCS and the ascending part of the IPS were not independent. The descending part of the IPS shifts to the superior occipital sulcus. In our study, we considered only the horizontal and descending parts as segments of the IPS, similar to Ono et al. ${ }^{8}$ We considered the inferior end of the descending part of the IPS to be the point at which the IPS crossed the theoretical border of the parietal and occipital lobes; thus, it was difficult to identify the inferior end of the descending part of the IPS.

The results from our studies of sulcal connections were in good agreement with those in previous reports by Ono et al. ${ }^{8}$ Other studies with specimens and 2D MR images have examined the connection of the postCS and the IPS and found that the frequency with which the postCS connected with the IPS ranged between $65.8 \%$ and $78 \%$.,11 Similar to findings by Ono et $\mathrm{al},{ }^{8}$ we found that the sulcal connections of the postCS and Sy varied between the right and left sides. ${ }^{8}$ We concluded

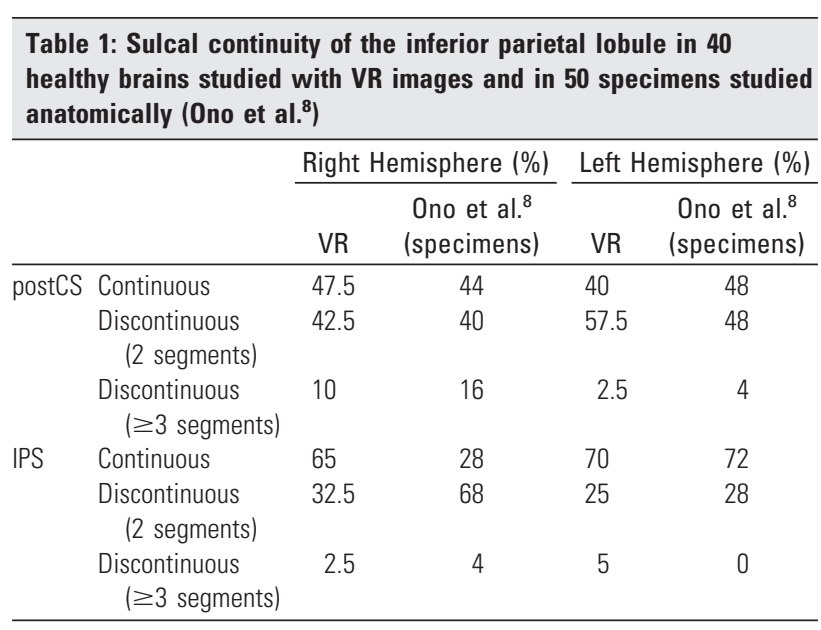

Note:- postCS indicates postcentral sulcus; VR, volume rendering; IPS, intraparietal sulcus.

\begin{tabular}{|c|c|c|c|c|}
\hline \multirow{2}{*}{$\begin{array}{l}\text { postCS } \\
\text { Connected } \\
\text { with }\end{array}$} & \multicolumn{2}{|c|}{ Right Hemisphere (\%) } & \multicolumn{2}{|c|}{ Left Hemisphere (\%) } \\
\hline & VR & $\begin{array}{c}\text { Ono et al }{ }^{8} \\
\text { (specimens) }\end{array}$ & VR & $\begin{array}{c}\text { Ono et al }{ }^{8} \\
\text { (specimens) }\end{array}$ \\
\hline IPS & 72.5 & 64 & 75 & 72 \\
\hline Sy & 52.5 & 68 & 30 & 48 \\
\hline
\end{tabular}

Note:-IPS indicates the anterior end of the intraparietal sulcus; Sy, Sylvian fissure the posterior ascending ramus of the Sylvian fissure).

that our VR images depict the sulcal continuity and connections of the IPL accurately.

In the right hemisphere, the frequency of different gyral patterns of the IPL agreed with previous reports by Naidich et al. ${ }^{10}$ In the left hemisphere, no preAG pattern was seen compared with previous studies, which found the preAG pattern present in $28 \%$ of cases. ${ }^{10}$ The pattern in which additional gyri were positioned superior to the SMG, between the postCG and the AG, was called the preAG pattern by Naidich et al. ${ }^{10}$ In contrast, we defined this pattern either as preSMG or as not identifiable because we classified the IPL according to the order of gyri proceeding from anterior to posterior.

We also analyzed the IPL in 20 human fixed specimens. Results from VR images agreed with findings from these specimens in the right hemisphere. In the left hemisphere, the frequency of typical pattern on VR (35\%) was slightly higher than that in the specimens $(20 \%)$, and the frequency of the preSMG pattern in VR (17.5\%) was slightly lower than that in the specimens (35\%). These differences may be explained by differences in sample size between the 2 groups.

We identified other patterns in addition to typical, preSMG, and preAG, classifying them according to the gyral array as follows: 1) a case with additional gyri positioned both anterior and posterior to the SMG was assumed to be preSMG plus preAG, and 2) a case without a clear boundary between the SMG and the AG was assumed to be SMG plus AG. Figure 4 shows the schema and the cases on VR and specimens of these patterns. In the left hemisphere, SMG plus AG reached $37.5 \%$ and $40 \%$ on VR and specimens, respectively. An SMG plus AG pattern is assumed to be 1 type of IPL. 
Table 3: Frequency of gyral patterns of inferior parietal lobule in 40 healthy brains studied with VR images, in 20 specimens studied anatomically, and in $\mathbf{5 0}$ specimens studied anatomically by Naidich et $\mathbf{a l}^{10}$

\begin{tabular}{|c|c|c|c|c|c|c|}
\hline & \multicolumn{3}{|c|}{ Right Hemisphere (\%) } & \multicolumn{3}{|c|}{ Left Hemisphere (\%) } \\
\hline & VR & Specimens & $\begin{array}{l}\text { Naidich et al }^{10} \\
\text { (specimens) }\end{array}$ & VR & Specimens & $\begin{array}{c}\text { Naidich et al }{ }^{10} \\
\text { (specimens) }\end{array}$ \\
\hline Typical & 42.5 & 45 & 48 & 35 & 20 & 28 \\
\hline preSMG & 2.5 & 5 & 4 & 17.5 & 35 & 16 \\
\hline preAG & 17.5 & 20 & 16 & 0 & 5 & 28 \\
\hline Others & 37.5 & 30 & $32^{*}$ & 47.5 & 45 & $28^{*}$ \\
\hline (preSMG + preAG) & (0) & (15) & - & (2.5) & (0) & - \\
\hline$(S M G+A G)$ & $(12.5)$ & (10) & - & (37.5) & (40) & - \\
\hline (Pattern not identifiable) & (25) & (5) & - & (7.5) & (5) & - \\
\hline
\end{tabular}

Note:-preSMG indicates additional gyri intercalated between the postcentral sulcus (postCS) and the supramarginal gyrus (SMG); preAG, additional gyri positioned between the SMG and the angular gyrus (AG).

${ }^{*}$ Others were described as pattern not identifiable in the report by Naidich et $a^{10}$
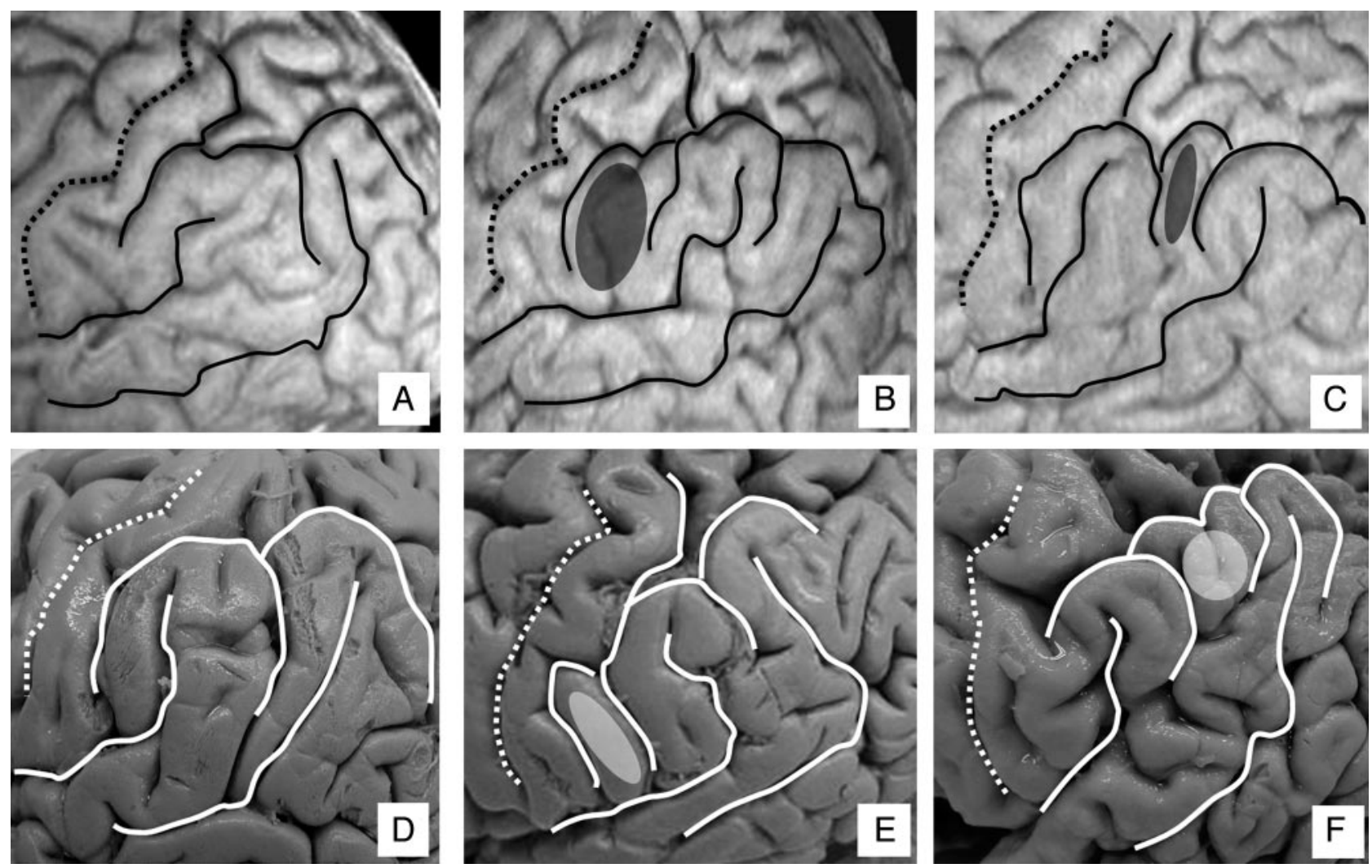

Fig 3. The IPL on VR images and with specimens. $A$ and $D$, Typical pattern. $B$ and $E$, PreSMG pattern. $C$ and $F$, PreAG pattern. $A$ through $C$ indicate VR images; $D$ through $F$, specimens. The additional gyri are colored.

Consistent with previous reports, the typical pattern was present less frequently on the left compared with the right side, both in our VR group and in our specimen group. The left hemisphere is often the dominant hemisphere for language, and it is important to recognize that the left hemisphere can show variable morphologic features. ${ }^{17,18}$

One limitation of our study was that the VR images of healthy volunteers were compared with those from previous reports and with specimens, which was insufficient to test the hypothesis that VR images can depict the topography of the IPL accurately. However, there are currently no techniques to observe the living brain other than VR images, and there were no remarkable differences between the results on VR images and specimens. We conclude that VR images can accurately display the topography of the IPL. Although there are some differences between our results and previous reports, these may be due predominantly to the variable morphologic features of the IPL.

\section{Conclusions}

Previously, the entire topography of the IPL could be observed only in postmortem specimens. VR images generated from high-resolution 3D MR imaging data allowed us to observe the entire IPL in living subjects. We compared the topography of the IPL on VR images with past reports describing postmortem specimens and believe that VR images can accurately display the topography of the IPL. In addition, we discovered new patterns of the gyral formations of the IPL. With only 2D transaxial and/or sagittal images, it is difficult to understand the topography of the entire IPL; thus, VR imaging is a very 

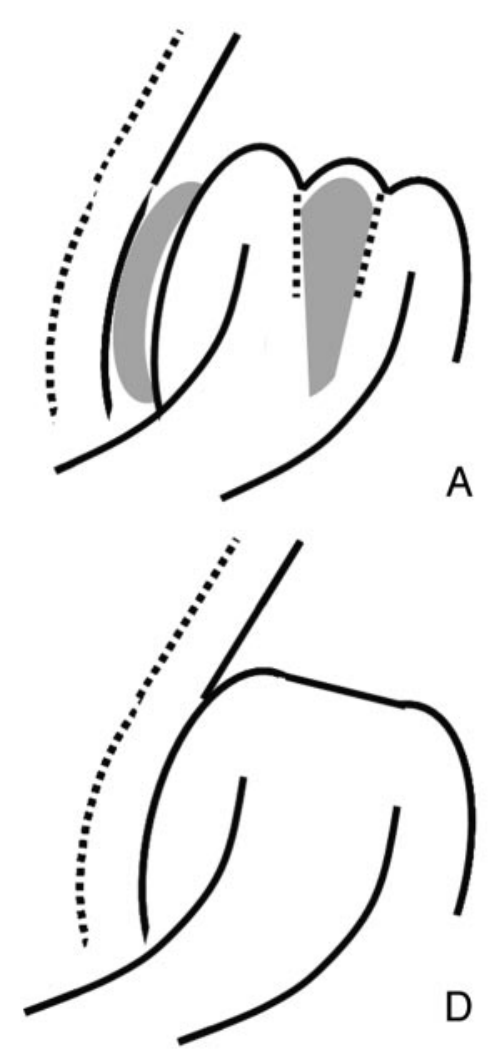
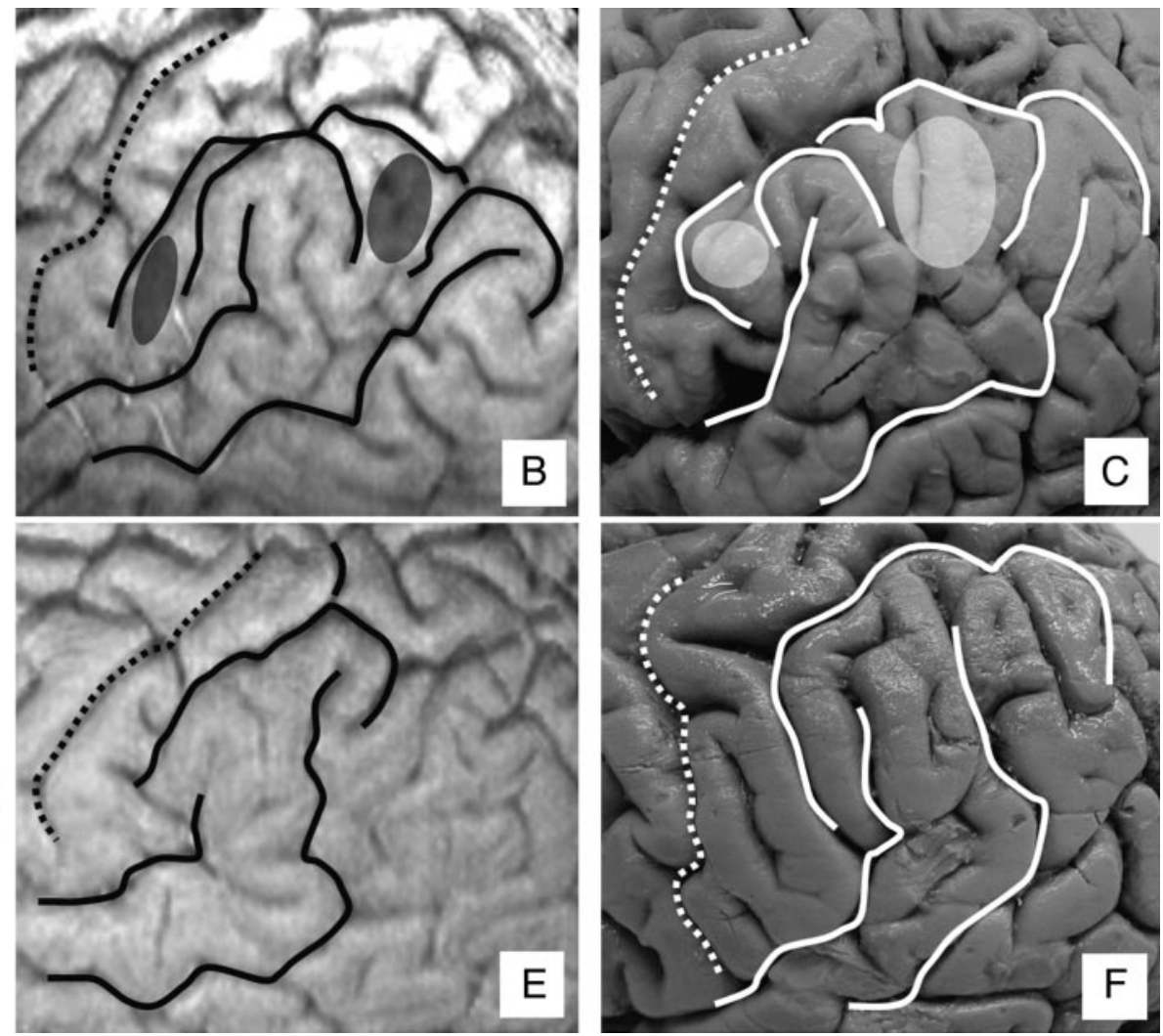

Fig 4. Schematic (left), VR images (middle), and specimens (right) of patterns of the preSMG plus preAG pattern and the SMG plus AG pattern. A-C, PreSMG plus preAG pattern. The additional gyri (gray tissue) positioned both anterior and posterior to the SMG. D-F, SMG plus AG pattern. There is no clear boundary between the SMG and the AG.

useful, noninvasive technique to observe the IPL in patients. VR imaging can contribute to the investigation of functional localization.

\section{References}

1. Gerstmann J. Syndrome in finger agnosia, disorientation for right and left, agraphia, and acalculia. Arch Neurol Psychiatry 1940;44:398-408

2. Heimburger RF, Demyer W, Reitan RM. Implications of Gerstmann's syndrome. J Neurol Neurosurg Psychiatry 1964;27:52-57

3. Roux FE, Boetto $S$, Sacko $O$, et al. Writing, calculating, and finger recognition in the region of the angular gyrus: a cortical stimulation study of Gerstmann syndrome. J Neurosurg 2003;99:716-27

4. Swisher JD, Halko MA, Merabet LB, et al. Visual topography of human intraparietal sulcus. J Neuroscience 2007;27:5326-37

5. Culham JC, Kanwisher NG. Neuroimaging of cognitive functions in human parietal cortex. Curr Opin Neurobiol 2001;11:157-63

6. Grefkes $C$, Fink GR. The functional organization of the intraparietal sulcus in humans and monkeys. J Anat 2005;207:3-17

7. Orban GA, Claeys K, Nelissen K, et al. Mapping the parietal cortex of human and non-human primates. Neuropsychologia 2006;44:2647-67

8. Ono M, Kubik S, Abernathy CD. Atlas of the Cerebral Sulci. New York: Thieme; 1990:62-74
9. Connolly CJ. External Morphology of the Primate Brain. Springfield, Illinos Thomas; 1950:204-22

10. Naidich TP, Valavanis AG, Kubik S. Anatomic relationships along the lowmiddle convexity: part 1-normal specimens and magnetic resonance imaging. Neurosurgery 1995;36:517-32

11. Ebeling U, Steinmetz H. Anatomy of the parietal lobe: mapping the individual pattern. Acta Neurochir 1995;136:8-11

12. Steinmetz H, Ebeling U, Huang Y, et al. Sulcus topography of the parietal opercular region: an anatomic and MR study. Brain Language 1990;38: 515-33

13. Levin DN, Hu X, Tan KK, et al. Surface of the brain: three-dimensional MR images created with volume rendering. Radiology 1989;171:277-80

14. Levoy M. Display of surfaces from volume data. IEEE Computer Graphics Applications 1988;8:29-37

15. Miki H, Hiratsuka Y, Kikuchi K, et al. High-resolution three-dimensional MR techniques for topographic analysis of frontal operculum. ISMRM 13th Scientific Meeting, 7-13 May 2005; Miami Beach, Fla

16. Duvernoy HM. The Human Brain. Surface, 3-D Sectional Anatomy and MRI New York: Springer-Verlag; 1991:10-11

17. Hamani C. Language dominance in the cerebral hemispheres. Surg Neurol 1997;47:81-83

18. Foundas AL, Leonard CM, Glimore R, et al. Planum temporale asymmetry and language dominance. Neuropsychologia 1994;32:1225-31 